\title{
Ground state and normal-mode spectra of a two-dimensional system of dipole particles confined in a parabolic trap
}

\author{
F. F. Munarin, ${ }^{1,2, *}$ W. P. Ferreira, ${ }^{2}$ G. A. Farias, ${ }^{2}$ and F. M. Peeters ${ }^{1, \dagger}$ \\ ${ }^{1}$ Department of Physics, University of Antwerp, Groenenborgerlaan 171, B-2020 Antwerpen, Belgium \\ ${ }^{2}$ Departamento de Física, Universidade Federal do Ceará, Caixa Postal 6030, Campus do Pici, 60455-760 Fortaleza, Ceará, Brazil
}

(Received 3 June 2008; published 17 September 2008)

\begin{abstract}
The ordered configurations of a monolayer of interacting magnetic dipoles confined in a circular parabolic potential are investigated as a function of the dipole moment of the particles. Despite the circular confinement, we find very asymmetric ordered structures like chains and Y-shaped configurations when a magnetic field is applied parallel to the plane of the particles. The normal-mode spectrum of the particles and its dependence on the magnetic field and the strength of the dipole moment of the particles are studied. The vibrational and rotational modes of the spectrum, which are associated with the stability of the system, are investigated in detail. The number of particles is varied and we found different ordering of the particles for different values of the dipole moment and the magnetic field. A ring structure with a large number of particles is observed for high values of the dipole moment of the particles.
\end{abstract}

DOI: 10.1103/PhysRevE.78.031405

PACS number(s): 82.70.Dd, 47.65.Cb

\section{INTRODUCTION}

The structure and collective behavior of magnetic colloidal particles exhibit considerable challenges to condensed matter physics with possible applications in various disciplines. For biomedical applications, these microspheres are paramagnetic particles which become magnetic in the presence of an external magnetic field [1]. They are frequently encountered in diverse physical experiments $[2,3]$. On the other hand, colloidal ferrofluids are dipolar fluids consisting of spherical ferromagnetic particles having a permanent magnetic dipolar moment present in diverse systems in medicine [4], which have been studied theoretically [5], and are utilized in many industrial applications [6,7]. The structural behavior of ferrofluids is mainly governed by the dipolar interaction, leading to a self-assembling of particles into a variety of magnetic equilibrium structures such as rings (flux closure), wormlike, branched dipole chains, and crystalline lattices $[2,8-10]$. Recently, there has been an increased experimental interest in colloidal ferrofluids due to the improvement of synthetic methods and the use of highresolution transmission electron microscopy [11-13]. For instance, field-induced two-dimensional structures in a ferrofluid of magnetic nanoparticles with a permanent magnetic dipole moment were analyzed on the single-particle level by in situ cryogenic transmission electron microscopy (cryoTEM) [14].

The different ordered structures and the phase behavior of colloidal ferrofluids have been studied in two $[9,14,15]$ and three dimensions $[2,16,17]$. However, the ordering of such systems in reduced dimensions is not necessarily equivalent to that of the related three-dimensional system. Moreover, calculations on two-dimensional dipolar systems are more directly relevant for the common experimental situation than those on bulk systems, because structural characterization of

\footnotetext{
*munarin@fisica.ufc.br

†francois.peeters@ua.ac.be
}

highly aggregated ferrofluids are largely carried out on thin films or on monolayers [11-13].

Recently, special attention was paid to finite-size systems of interacting particles subjected to parabolic [18] and Coulomb potentials [19]. Several experiments have been performed on dust plasma where Coulomb particles move in a plane and are confined in a parabolic trap. Both their ground state configuration [20] and the normal modes [21] were measured. Another experimental system is the one of paramagnetic colloidal particles in a two-dimensional circular cavity with varying depth that simulates a parabolic confinement potential [22]. Theoretic studies on such confined systems showed that the ordered structure of the ground state influences the dynamic of the particles [18] and the melting process [23]. In ferrofluids, Duncan and Camp [15] recently studied experimentally a quasi-two-dimensional dipolar fluid and observed characteristic frequencies of the single-particle orientational and translational motion for different values of the dipole moment of the particles which induce different kinds of structural arrangements of particles. In colloidal systems, clusters that are stabilized only by the magnetic interaction between the particles were studied recently [24]. It was found that the arrangement of the hard core particles within the cluster is imposed by magnetic frustration.

Motivated by the increasing experimental and technological interest in dipolar systems, we study in this paper the equilibrium configurations $(T=0)$ of a two-dimensional system of dipolar particles confined by a parabolic potential. We explore the dependence of the ground state configuration on the strength of the permanent magnetic dipole moment of the particles. The particles spontaneously self-assemble into a variety of interesting structures. If a magnetic field is applied parallel to the plane of the particles a diverse range of possible configurations are found (e.g., ring, chain, and Y-shaped structures). In addition, we investigate the normal-mode frequencies of the ground state configurations with and without the presence of an external magnetic field. The vibrational and rotational components of the mode associated with the lowest nonzero frequency are found and they show a strong 
dependence on the external magnetic field and the size of the dipole moment of the particles. When the number of particles is varied, we find different configurations for different sizes of the dipole moment and magnetic field.

This paper is organized as follows. In Sec. II, we describe the mathematical model and summarize the numerical approach to obtain the ground state configurations of the system. Section III A contains the results for the dependence on the dipolar moment with and without the influence of a magnetic field parallel to the plane of the particles. Section III B presents an analysis of the spatial structure of the ground state and its normal modes for different magnetic field intensities. The system structure and normal modes for different numbers of particles with and without the presence of a magnetic field are investigated in Sec. III C. Our conclusions are presented in Sec. IV.

\section{MODEL}

Our system consists of a monolayer of $N$ particles interacting with each other via a dipole-dipole pair potential. The particles are kept together by a parabolic potential centered at the origin. We will introduce a preferential direction in the problem by applying a magnetic field parallel to the confinement plane. The case of a perpendicular magnetic field was considered before and leads to results that are similar to the Coulomb and Yukawa type of interparticle interaction $[25,26]$. The potential energy of our system is given by

$$
\begin{aligned}
H= & \sum_{i}\left(\frac{1}{2} m \omega_{0}^{2} r_{i}^{2}-\boldsymbol{\mu}_{i} \cdot \mathbf{B}\right)+\sum_{i<j=1}^{N}\left[4 \varepsilon\left(\frac{\sigma}{r_{i j}}\right)^{12}+\frac{\boldsymbol{\mu}_{i} \cdot \boldsymbol{\mu}_{j}}{r_{i j}^{3}}\right. \\
& \left.-\frac{3\left(\boldsymbol{\mu}_{i} \cdot \mathbf{r}_{i j}\right)\left(\boldsymbol{\mu}_{j} \cdot \mathbf{r}_{i j}\right)}{r_{i j}^{5}}\right]
\end{aligned}
$$

where $\varepsilon$ is an energy parameter which characterizes the short-range repulsion of the particles preventing them from coalescing in a single point, $\boldsymbol{\mu}_{i}$ is the dipole vector on particle $i, \mathbf{r}_{i j}$ is the interparticle separation vector between particles $i$ and $j, r_{i} \equiv\left|\mathbf{r}_{i}\right|$ is the distance of the $i$ th particle from the center of the parabolic confinement, $m$ is the mass, and $\sigma$ is the diameter of the colloids. In order to reveal the important parameters of the system, it is convenient to define $\mu^{*}$ $=\sqrt{\mu^{2} / \varepsilon \sigma^{3}}$ and to use $\varepsilon$ for the unit of energy, $B_{0}=\sqrt{\varepsilon / \sigma^{3}}$ for the unit of magnetic field, and $\sigma$ (a measure of the radius of the colloids) for the characteristic length scale in this system. In so doing, we obtain the potential energy (1) in dimensionless form,

$$
\begin{aligned}
H= & \sum_{i}\left(\alpha r_{i}^{2}-\boldsymbol{\mu}_{i}^{*} \cdot \mathbf{B}\right)+\sum_{i<j=1}^{N}\left[4\left(\frac{1}{r_{i j}}\right)^{12}+\frac{\boldsymbol{\mu}_{i}^{*} \cdot \boldsymbol{\mu}_{j}^{*}}{r_{i j}^{3}}\right. \\
& \left.-\frac{3\left(\boldsymbol{\mu}_{i}^{*} \cdot \mathbf{r}_{i j}\right)\left(\boldsymbol{\mu}_{j}^{*} \cdot \mathbf{r}_{i j}\right)}{r_{i j}^{5}}\right],
\end{aligned}
$$

which indicates that the system depends on the number of particles $N$, the relative strength of the confinement potential $\alpha=m \omega^{2} \sigma^{2} / 2 \varepsilon$, the dipolar moment of the particles $\boldsymbol{\mu}_{i}^{*}$, and the external magnetic field $\mathbf{B}$. The interaction energy be- tween the dipole $i$ and the applied magnetic field is described by the term $-\boldsymbol{\mu}_{i}^{*} \cdot \mathbf{B}$. In our study, we took $\alpha=1$ and applied the magnetic field along the $x$ axis, i.e., $\mathbf{B}=(B, 0,0)$.

The ground state configurations $(T=0)$ of the present system were obtained using the Monte Carlo annealing simulation technique followed by a Newton optimization procedure [27]. The annealing simulation method was used in order to ensure that the obtained energy was close to the global minimum. To check if a configuration is stable, we calculated, using the Householder diagonalization technique, the eigenvalues of the dynamical matrix

$$
H_{\alpha \beta, i j}=\frac{\partial^{2} H}{\partial r_{\alpha, i} \partial r_{\beta, j}},
$$

where $\alpha, \beta=x, y$ and $\theta$. The $i, j$ indices indicate the particle number and $\theta$ is the angle between their dipole moments. From all the stable states obtained, the one with the lowest energy was taken as the ground state.

\section{RESULTS}

\section{A. Dependence on dipole moment $\mu^{*}$}

In the following, we analyze, as an example, a system composed of $N=19$ dipolar particles as a function of the dipole moment of the particles $\mu^{*}$ with and without the presence of a magnetic field $B$. Initially, the ground state configuration is obtained in the absence of an external magnetic field where the minimum energy structure is a consequence of the strength of the interparticle interaction. After that, the $B \neq 0$ case will be considered.

\section{Zero magnetic field}

To study the structure of the ground state of the system, Fig. 1(a) shows the distance of the $N=19$ particles with respect to the center of the confinement potential as a function of $\mu^{*}$. We clearly see five structural transitions indicated by the vertical dashed lines between the different configurations which are presented in Figs. 1(c)-1(f). For $\mu^{*} \approx 0$ [Fig. 1(c)], the configuration consists of shells with a local hexagonal arrangement of particles as observed experimentally in infinite systems of dipolar particles [28]. The hexagonal ground state structure for $\mu^{*}=0$ is a specific feature of the balance between the parabolic confinement potential and the isotropic $r^{-12}$ repulsive interparticle interaction [Eq. (2)]. When the dipole moment is increased from $\mu^{*}=0$ to $\mu^{*} \approx 1.6$ (region I), the system configuration remains unaffected but now the dipole moments of the particles are oriented in a kind of spiral shape as indicated by the dashed line in Fig. 1(c). This behavior reveals the angular dependence between the dipole moments of the particles which attempts to align them.

At $\mu^{*} \approx 1.6$ (region II), the dipolar interaction starts to influence the ground state configuration which changes to a transient configuration between a hexagonal-shaped and a ring structure. As can be seen in Fig. 1(d), one particle moved from the center of the system to the inner shell and another one moved from the inner shell to the outer one, forming a structure of noncircular rings. This configuration is a result of the in-plane dipole-dipole interaction which tends 


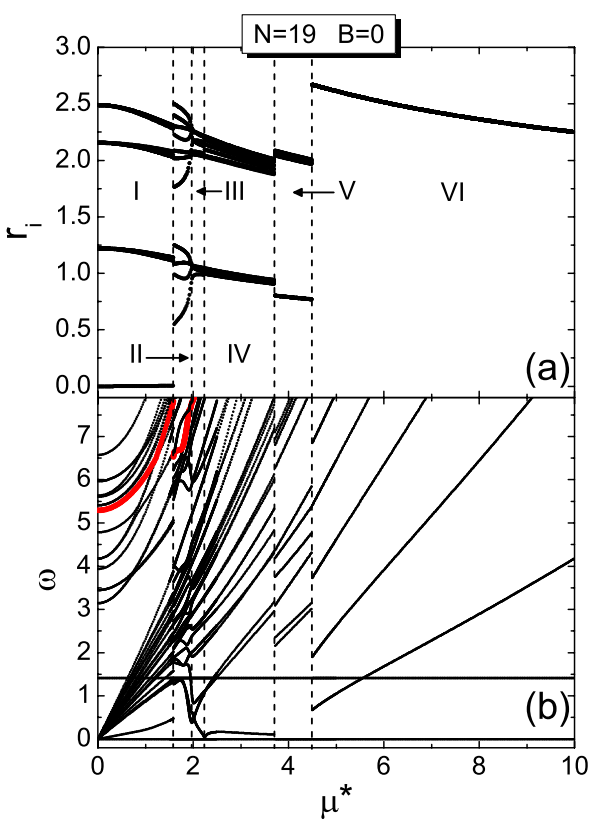

\begin{tabular}{|c|c|c|}
\hline$B=0$ & $B=0 \quad \mu^{*}=1.62$ & $B=\mu^{\prime}$ \\
\hline$\phi \theta \varnothing \varnothing \varnothing$ & $\begin{array}{ccccc}\theta & \theta & & \\
\theta & \theta & \theta & \\
(1) & \oplus & \theta & & \theta \\
\theta & \theta & \theta & \theta & \\
\theta & \theta & \theta & \end{array}$ & $\begin{array}{llll}\text { III } & \theta & \theta & \\
\theta & \theta & \theta & \theta \\
(1) & \oplus & & \theta \\
0 & \theta & \theta & \theta \\
& \theta & \theta & \theta\end{array}$ \\
\hline 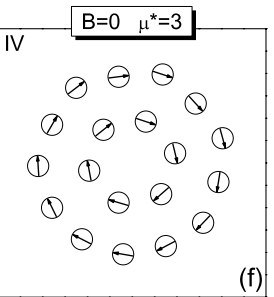 & 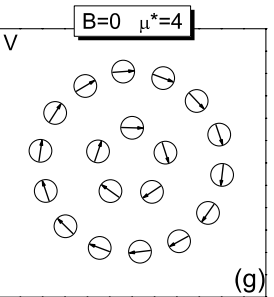 & $\operatorname{\theta }_{\theta \theta \theta \theta^{\theta \theta \theta}}^{\theta_{\theta}}$ \\
\hline
\end{tabular}

FIG. 1. (Color online) (a) Radial position and (b) normal-mode frequency of $N=19$ dipolar particles in zero magnetic field $B=0$ as a function of the dipole moment of the particles $\mu^{*}$. The associated breathing mode in $\mu^{*}=0$ is shown as a red line. (c) $-(\mathrm{g})$ The ground state configuration of the system for each region defined in (a). The dashed line shown in (c) is a guide to the eye.

to align the particles in a chain, but due to the parabolic confinement potential turns them into a ringlike structure. When $\mu^{*}$ is further increased, the attraction between the dipoles becomes sufficiently strong and as a consequence the system configuration changes continuously to two rings shown in Figs. 1(e) and 1(f) regions III and IV) through two structural transitions at $\mu^{*} \approx 1.97$ and $\mu^{*} \approx 2.2$, respectively. From Fig. 1(a) we see in region IV that the outer shell has a finite width and thus forms a nonperfect shell of particles. At $\mu^{*} \approx 3.7$ (region V), a clear ringlike structure is observed [Fig. 1(g)], but now with a different number of particles in each shell as compared to the structure in regions III and IV. The most symmetric configuration is obtained for $\mu^{*}>4.5$ when the system consists of a single ring [see Fig. 1(g)].
In Fig. 1(b), the normal-mode frequency (NMF) spectrum of the system in units of $\omega_{0} / \sqrt{2}$ is shown as a function of $\mu^{*}$. The same regions I-VI as previously defined in Fig. 1(a) are also indicated. The NMFs are sensitive to structural transitions, showing a discontinuous change when a first-order structural transition occurs, and a typical softening in one of the frequencies in the case of a second-order transition. In general, most of the NFMs increase with $\mu^{*}$. However, there are two special frequencies, namely, $\omega=0$ and $\sqrt{2}$, which remain constant for all values of $\mu^{*}$. These frequencies are associated with the rotation of the entire cluster around the center and the motion of the center of mass, respectively. Another special frequency [indicated as a red line in Fig. 1(b)], associated with the breathing mode of the particles $\left(\omega=5.29\right.$ for $\left.\mu^{*}=0\right)$, also increases as a function of $\mu^{*}$. This behavior is due to the fact that this frequency depends on the confinement and interaction potential [29] and as a consequence its frequency increases with the dipolar moment of the particles, $\mu^{*}$. In region I there are clearly two classes of normal modes: the low-frequency modes whose frequencies increase linearly with $\mu^{*}$ and a set of higher-frequency modes that increase quadratically with $\mu^{*}$. The normal-mode frequencies in regions II and III exhibit a rather complex behavior which is due to the gradual coalescence of particles into a ringlike structure [see Fig. 1(a)]. In regions IV, V, and VI the NMF increases roughly linearly with $\mu^{*}$ except for the lowest nonzero frequency.

Now we carefully analyze the behavior of the lowest nonzero frequency (LNF) mode and its associated oscillation, which is linked with the stability of the ground state configuration. In addition, this LNF mode plays an important role in the melting properties of the cluster [23,27]. In Fig. 2(a), the LNF of the cluster with $N=19$ particles and zero magnetic field is presented as a function of $\mu^{*}$. The same regions defined in Fig. 1(a) are indicated. However, new subregions separating different $\mu^{*}$ intervals in which the ground state configuration is similar, but the excited normal modes of vibration and/or rotation are different (regions IIa, IIb, IIc, VIa, and VIb), are also defined.

The vibrational motion of the particles in each region of Fig. 2(a) is shown in Figs. 2(b)-2(i). The arrows indicate the direction and its size represents the amplitude of oscillation. In general, the vibrational mode is different for each configuration. However, we find that distinct normal modes can be excited for the same minimum energy configuration. For example, in subregions IIa, IIb, and IIc, the configuration is given in Fig. 1(d), but different lowest normal modes are obtained [Figs. 2(c)-2(e), respectively]. In regions $\mathrm{V}$ and $\mathrm{VIb}$, it is observed that the LNF mode becomes the center of mass mode of the particles shown in Figs. 2(h)-2(j), respectively. Clearly, the LNF mode is tunable by $\mu^{*}$, and, as a consequence, also the stability of the cluster. Based on a recent study [23], this fact could indicate that $\mu^{*}$ also influences the melting temperature of the system.

Although the LNFs are different in regions III and IV [ see Fig. 2(a)], the normal modes in both regions are rather similar [Figs. 2(f) and 2(g)]. The understanding of the change in LNF behavior is complicated by the fact that the LNF is also associated with a rotational component of the individual dipoles. In order to analyze the associated lowest nonzero ro- 


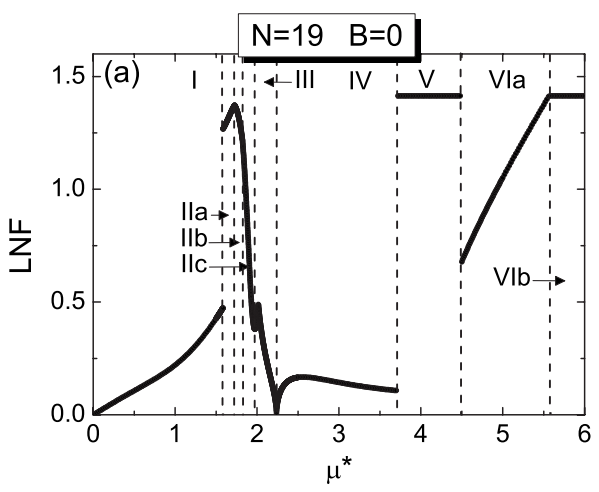

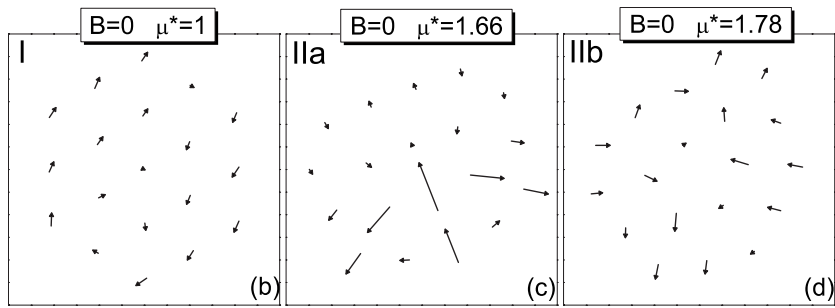
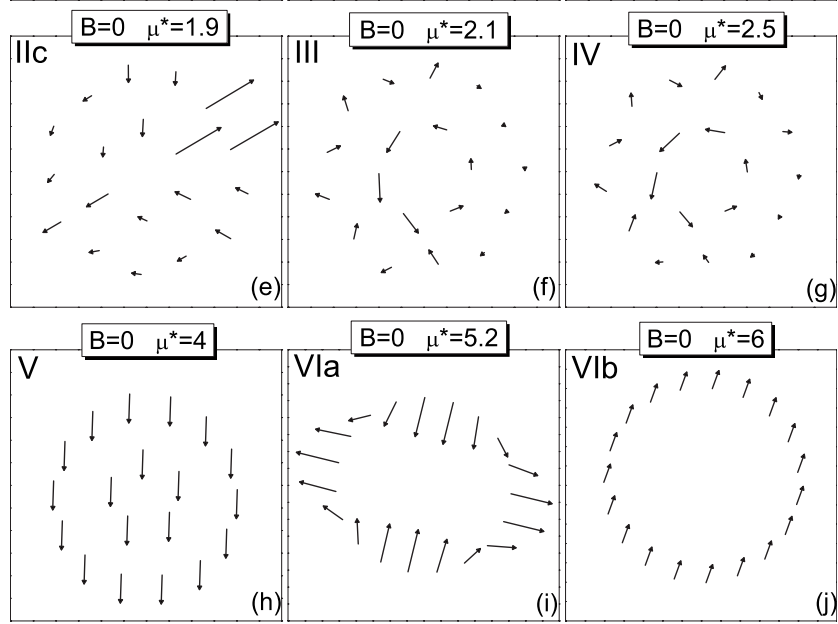

FIG. 2. (a) Lowest nonzero frequency (LNF) as a function $\mu^{*}$ for system with $N=19$ particles and $B=0$. (b)-(j) Normal modes of vibration for each subregion defined in (a).

tational mode, Figs. 3(a) $-3(\mathrm{~g})$ show the rotational mode of each dipolar particle for all regions defined in Fig. 2(a). The rotational mode is indicated by colors inside the circles representing the direction and amplitude of oscillation of each particle. The arrows indicate the orientation of the dipoles. When the system has a hexagonal structure (region I), we observe that the direction of oscillation of each particle are symmetric with respect to the center of the confinement potential [Fig. 3(a)]. Specifically, we observe that two particles of the inner shell are oscillating clockwise (light blue circles) while the other two oscillate counter clockwise (yellow circles). The central particle in Fig. 3(a) rotates counterclockwise with a large angular amplitude in order to conserve the total angular moment of the system where there are few particles oscillating with small angular amplitude (green particles).

Figures 3(a)-3(i) show that the rotational modes of the particles are different for each region of Fig. 2(a). It is also observed that there is no rotation of particles in regions $\mathrm{V}$

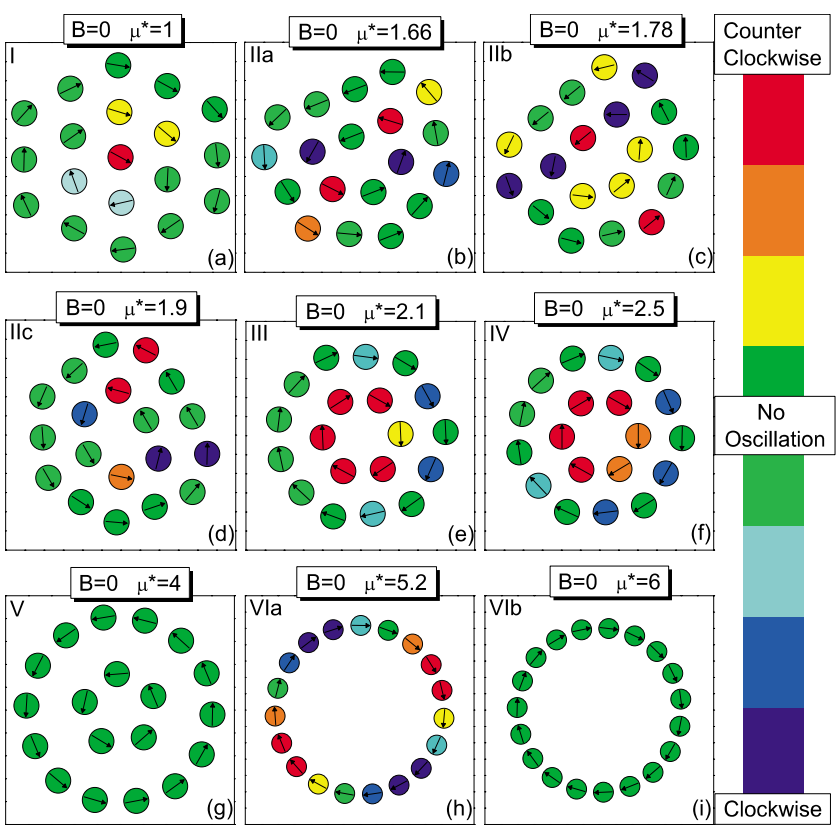

FIG. 3. (Color online) Rotational component of the LNF mode for the system with $N=19$ particles and $B=0$. In each figure the subregions defined in Fig. 2(a) are indicated. The color scale gives the amplitude and the direction of rotation of the particles.

and VIb [Figs. 3(g) and 3(i)] where the modes are associated with the motion of the center of mass of the system. In addition, the rotational component of the LNF reveals a very interesting characteristic in Fig. 2(a). As can be seen, the rotational modes of particles in the regions III and IV [Figs. 3(e) and 3(f)] are different although the configuration and the vibrational components of the mode are similar in these regions. This fact indicates that the second-order structural phase transition of the system between the regions III and IV is exclusively due to the change of the rotational mode of the particles and therefore is essential in the study of the stability of our dipolar system.

\section{In the presence of a magnetic field}

In the following we present the dependence of the configuration and the normal modes of $N=19$ particles on $\mu^{*}$ in the presence of a magnetic field $B$ applied along the $x$ axis. The magnetic field introduces a preferential direction into the problem which will tend to break the circular symmetry of the ordered structures. Here, we fix $B$ and vary $\mu^{*}$. In Fig. 4(a) we show the radial position of $N=19$ particles with respect to the center of the confinement potential as a function of $\mu^{*}$ for $B=5$. The system exhibits several structural phase transitions as a function of $\mu^{*}$ which are indicated in Fig. 4(a) by vertical dashed lines. Such lines separate regions with different ground state configurations shown in Figs. 4(c)-4(n).

For small values of $\mu^{*}$ (region I), the particles are arranged in the same configuration as observed in zero magnetic field [see Fig. 1(c)] but now all dipoles are oriented in the same direction, i.e., parallel to the magnetic field [Fig. $4(\mathrm{c})]$. At $\mu^{*} \approx 1.2$ (region II), the system experiences a first 

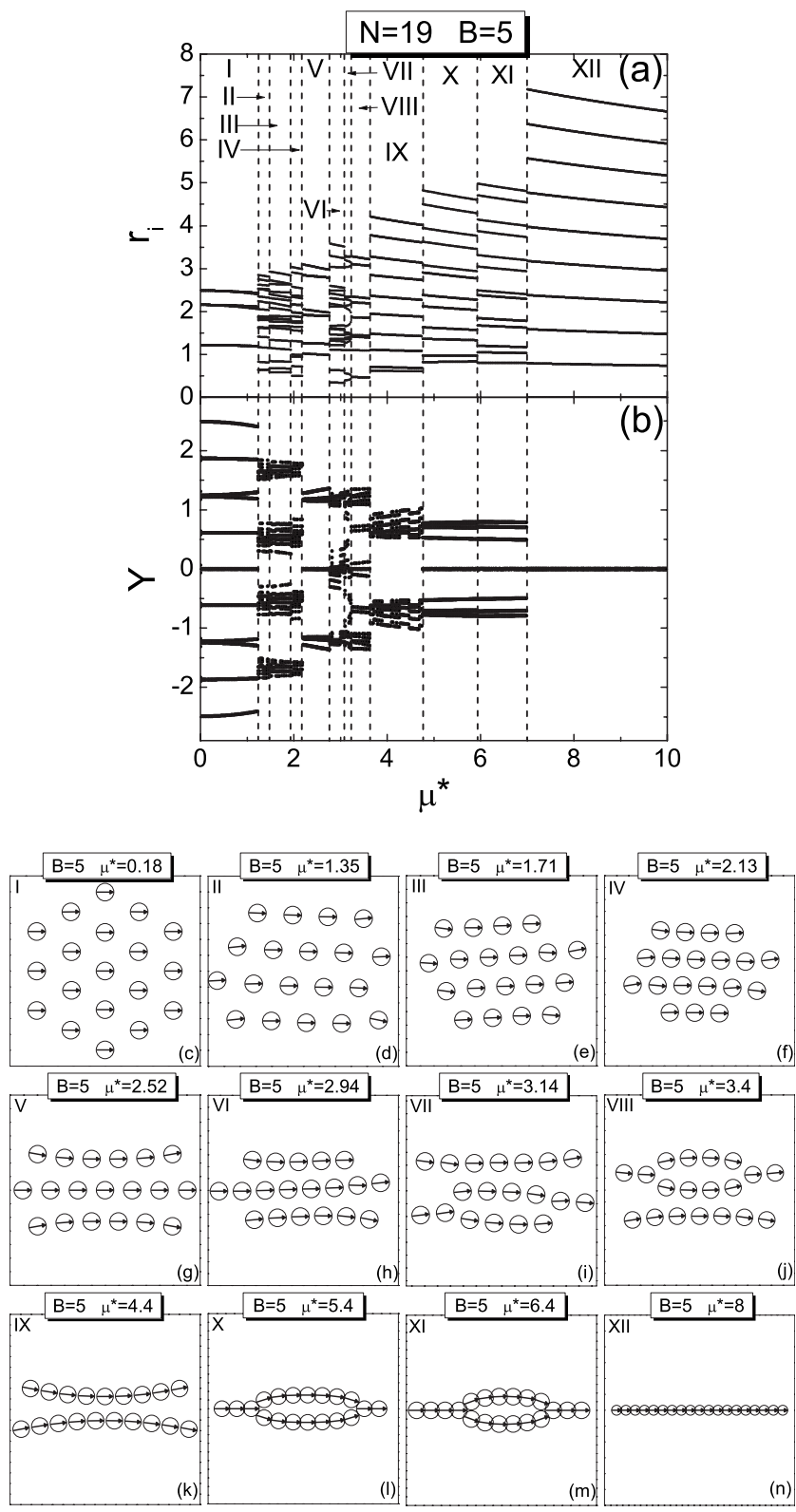

FIG. 4. (a) Radial position and (b) distance of each particles with respect to the $x$ axis of $N=19$ dipolar particles for $B=5$ as a function of dipole moment of particles $\mu^{*}$. (c)-(n) The ground state configuration of system for each region defined in (a).

structural phase transition and changes from a hexagonal to a four-line configuration as shown in Fig. 4(b). Notice that the chains are parallel to the $x$ axis, which means that the magnetic field adds an additional one-dimensional confinement to the parabolic potential. In order to better observe the symmetry of the system with respect to the $B$ direction, we present in Fig. 4(b) the distance of each particle with respect to the $x$ axis as a function of $\mu^{*}$. With further increase of $\mu^{*}$, we observe that particles of the outer chains (with respect to the $x$ direction) successively move to the inner chains, as a consequence decreasing the number of particles in the outer chains after each structural phase transitions. These transitions can be recognized by jumps in Figs. 4(a) and 4(b).
At $\mu^{*} \approx 3.1$ (region VII), the system turns into an unexpected configuration formed by the quasi-two-lines of particles shown in Figs. 4(i) and 4(j). These configurations are composed of a line of particles and a chain composed by a mixture of one- and two-lines, that is, the system presents a local splitting of a single line into two which we will call a 2Y-shaped configuration. The Y-shaped configuration was analyzed and observed in diverse theoretical and experimental studies in dipole particle systems [8,14,30,31], but here it appears as two connected Y-shaped configurations. This structure is due to the influence of the confinement potential which compresses the system connecting two lines through two dipolar particles. The size of the 2 Y-shaped configuration decreases up to $\mu^{*} \approx 3.64$ when the system transits into two curved lines (region IX). At $\mu^{*} \approx 4.76$, the system transits to a 2 Y-shaped configuration before it exhibits the configuration composed by a line of particles for $\mu^{*}>7$. It is important to observe that the Y-shaped configuration appears when the number of chains decreases, i.e., between the configurations with three and two chains (regions VI and IX) and between two and one chains (regions IX and XII). This fact indicates that the appearance of the Y-shaped configuration is due to the high density of particles in the $B$ direction caused by the balance between the confinement potential and the magnetic field.

Figure 5(a) shows the corresponding frequencies of the normal modes where the vertical lines indicate the transitions as shown also in Fig. 4(a). As is apparent, the degeneracy in the frequency spectrum found in the zero-magnetic-field case is lifted due to the preferential direction introduced by the applied field. In general, most of the frequencies increase with increasing $\mu^{*}$, as was found also in the case with $B$ $=0$. Notice that the $\mu^{*}$-independent frequency $\omega=0$, which was previously associated with the rotation of the entire cluster, attains a value $\omega \neq 0$ when $\mu^{*} \neq 0$. This fact is a consequence of the breaking of the rotational invariance introduced by the magnetic field. The system still exhibits one mode with a frequency that is independent of $\mu^{*}$, namely, the center of mass mode $(\omega=\sqrt{2})$.

The lowest nonzero normal-mode frequency is shown in Fig. 5(b) as a function of $\mu^{*}$ for $B=5$. New subregions separating different $\mu^{*}$ intervals in which the ground state configuration is the same, but the excited normal modes of vibration and/or rotation are different (subregions IXa, IXb, XIIa, and XIIb), are also defined. It is important to note that the LNF value becomes different from zero for small values of $B$. This fact confirms that the magnetic field influences the system even when it has a hexagonal structure as the ground state configuration. The vibrational components of the LNF mode associated with each region of Fig. 5(b) are shown in Figs. 5(c)-5(p). Figure 5(c) shows the vibrational component of the LNF in the hexagonal structure (region I). Note that this mode differs from the one associated with the rotation of the entire system by one particle motion which is presented by the red arrow. Figures $5(\mathrm{c})-5(\mathrm{p})$ show that the vibrational components of the modes are different for each configuration presented in Figs. 4(c)-4(n), i.e., they change as a function of $\mu^{*}$. Nevertheless, the configuration in the regions IX and 

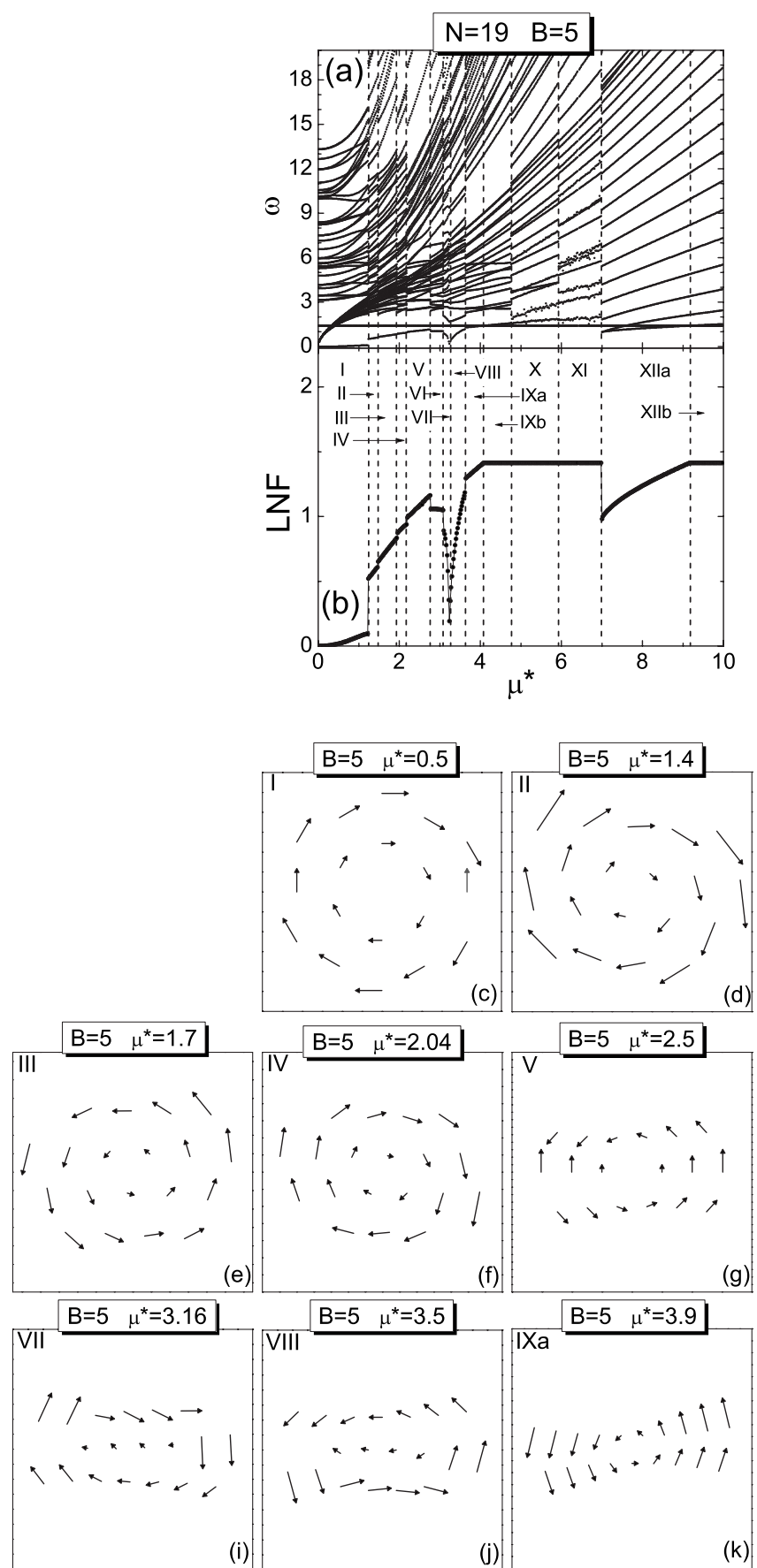

(g)
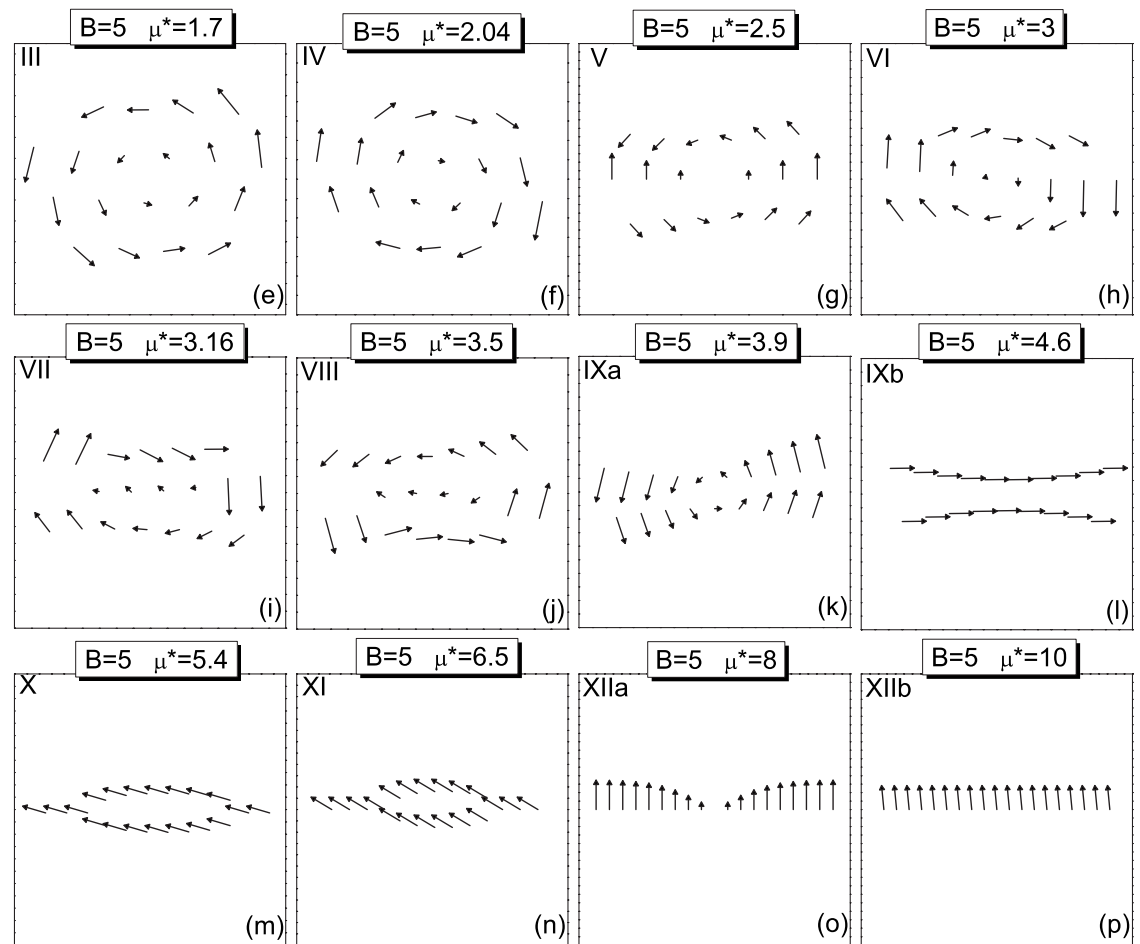

(k)
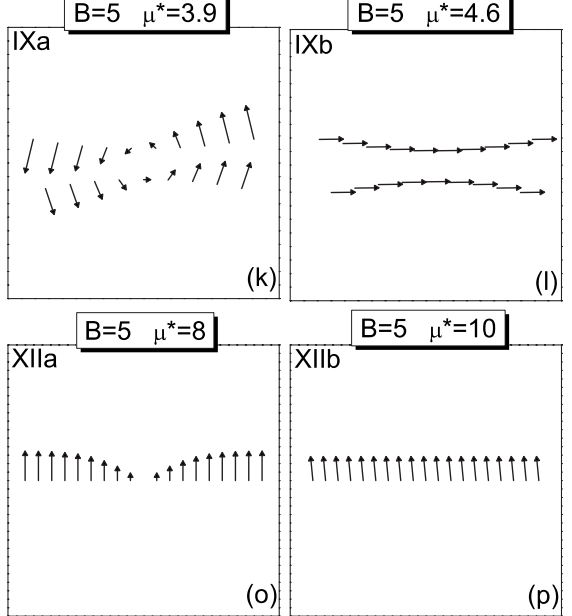

FIG. 5. (a) Normal-mode frequency and (b) lowest nonzero frequency of $N=19$ dipolar particles as a function of the dipole moment of the particles $\mu^{*}$ for $B=5$. (c)-(p) Normal modes of vibration for each subregion defined in (b).

XII of Fig. 5(b) present very different vibrational motions of particles. In addition, the center of mass mode becomes the LNF mode in the configuration of regions IX, X, XI, and
XIIb, where all particles are moving in the same direction with the same amplitude. Notice that in these regions the particles are moving in different directions, indicating that 


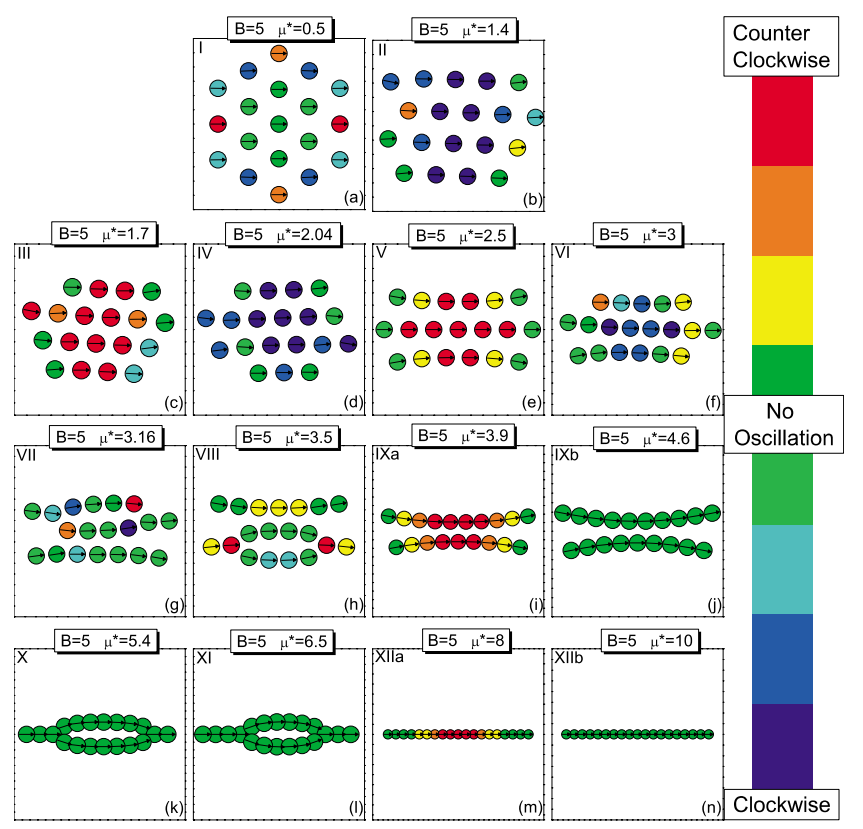

FIG. 6. (Color online) Normal modes of rotation for the system with $N=19$ particles and $B=5$. In each figure the subregions shown in Fig. 5(b) are indicated. The color scale indicates the amplitude and the direction of rotation of the particles.

the magnetic field $B$ does not influence the direction of vibration of particles.

In order to study the influence of the magnetic field in the rotational motion of particles, Figs. 6(a)-6(n) show the rotational components of the LNFs of particles in each region shown in Fig. 5(b). We observe that the particles of the hexagonal configuration present an interesting rotational behavior shown in Fig. 6(a). Unlike in the zero-magnetic-field case, the particles with the lowest rotational amplitude of oscillation are located in the center of the system. In addition, we observe that the particles with the same amplitude of oscillation are located symmetrically with respect to the directions parallel and perpendicular to the magnetic field. In the case of the chain configurations, regions II-VI, the particles with the highest amplitude of oscillation are located in the center of the chains. This scenario is clearly observed for the configuration of region $\mathrm{V}$ [Fig. 6(e)]. This behavior is due to the fact that the particles located in the center have more nearest neighbors than those in the extreme of the system and therefore there is more orientational coupling of the dipole moments. In this manner, the center particles rotate with the highest amplitude since they have more neighbor particles to align. This is clearer for the configurations of region $\mathrm{V}$ [Fig. 6(e)] because of its perfect symmetry with respect to the $x$ direction which is not observed in the configuration of Figs. 6(b)-6(d) and 6(f). This scenario is not observed when the system presents a 2 Y-shaped configuration shown in Figs. 6(g) and 6(h) (regions VII and VIII of Fig. 5(b)). Increasing $\mu^{*}$ further, we observe that the configuration corresponding to regions IX and XII in Fig. 5(b) presents different rotational modes for the same configuration, indicating that the rotational component of the LNF is strongly dependent on the $\mu^{*}$ value. It is important to notice that the angular amplitude of particles is zero when $\omega=\sqrt{2}$ (i.e., when $\left.\omega=\omega_{0}\right)$. This behavior is observed in the rotational motion of the system of regions IXb, X, XI, and XIIb which are shown in Figs. 6(j)-6(1) and 6(n), respectively.

\section{B. Dependence on magnetic field $B$}

In this section, we investigate the $N=19$ particle system with fixed dipole moment $\mu^{*}=5$ as a function of the magnetic field $B$ applied parallel to the $x$ axis of system. In Fig. 7 (a) we present the radial distance of each particle with respect to the center of the confinement potential as a function of $B$ for $\mu^{*}=5$. Despite the constant value of $\mu^{*}$, the system exhibits several structural phase transitions indicated by vertical dashed lines in Fig. 7(a). Figure 7(b) shows the distance of each particle with respect to the $x$ axis. As can be seen in Fig. 7(b), the symmetry of the configuration with respect to the $x$ axis increases as a function of $B$. The configurations in each region of Fig. 7(a) are shown in Figs. 7(c)-7(f). Up to $B \approx 2$ (region $\mathrm{I}$ ), the particles are ordered into a single ring [Fig. 7(c)]. At this $B$ value, the structure changes to an interesting nonsymmetric configuration which is a mixture of a single chain and an elliptic ring around it [Fig. 7(d)]. This nontrivial configuration [Fig. 7(d)] clearly shows the competition between the external forces, i.e., forces due to the magnetic field and the confinement potential.

In $B \approx 2.4$ (region III), the system transits to a 2 Y-shaped configuration, which was also observed in the previous section when the system was studied as a function of $\mu^{*}$. In $B$ $\approx 13$ (region IV), the system configuration changes to two parallel chains with different particles in each one. In order to minimize the energy of the system, the distances of each chain with respect to the $x$ axis are different as can be observed in region IV of Fig. 7(b). In this figure, we clearly observe an increase of the system symmetry with respect to the $x$ axis when the magnetic field is increased. In general, the dependence of the configuration on the magnetic field and on the dipole moment are similar, i.e., the system structure changes from a shell structure to chains as a function of $\mu^{*}$ for $B$ fixed, and vice versa. Nevertheless, the transient configurations found are different when we vary $\mu^{*}$ or $B$. In addition, we note that the final configuration of the system is composed of two chains [Fig. 7(f)] of particles instead of one [Fig. 4(n)]. This is due to the fact that the attraction between the particles is not large enough to form a single chain. Therefore, we can conclude that the value of the magnetic field $B$ guides the symmetry of the system with respect to the $B$ direction, while the dipole moment $\mu^{*}$ controls the number of chains parallel to it.

In Fig. 8(a), the frequencies of the normal modes of vibration and rotation of the system are shown as a function of $B$ for $\mu=5$. It is divided in the same regions as defined in Fig. 7(a). It is important to note that most frequencies do not increase further when the magnetic field $B$ is increased. On the other hand, the frequency associated with the rotation of the whole system, which is directly associated with the confinement potential, increases as a function of $B$, indicating that the magnetic field also acts as an effective confinement potential in one direction. The behavior of this frequency 

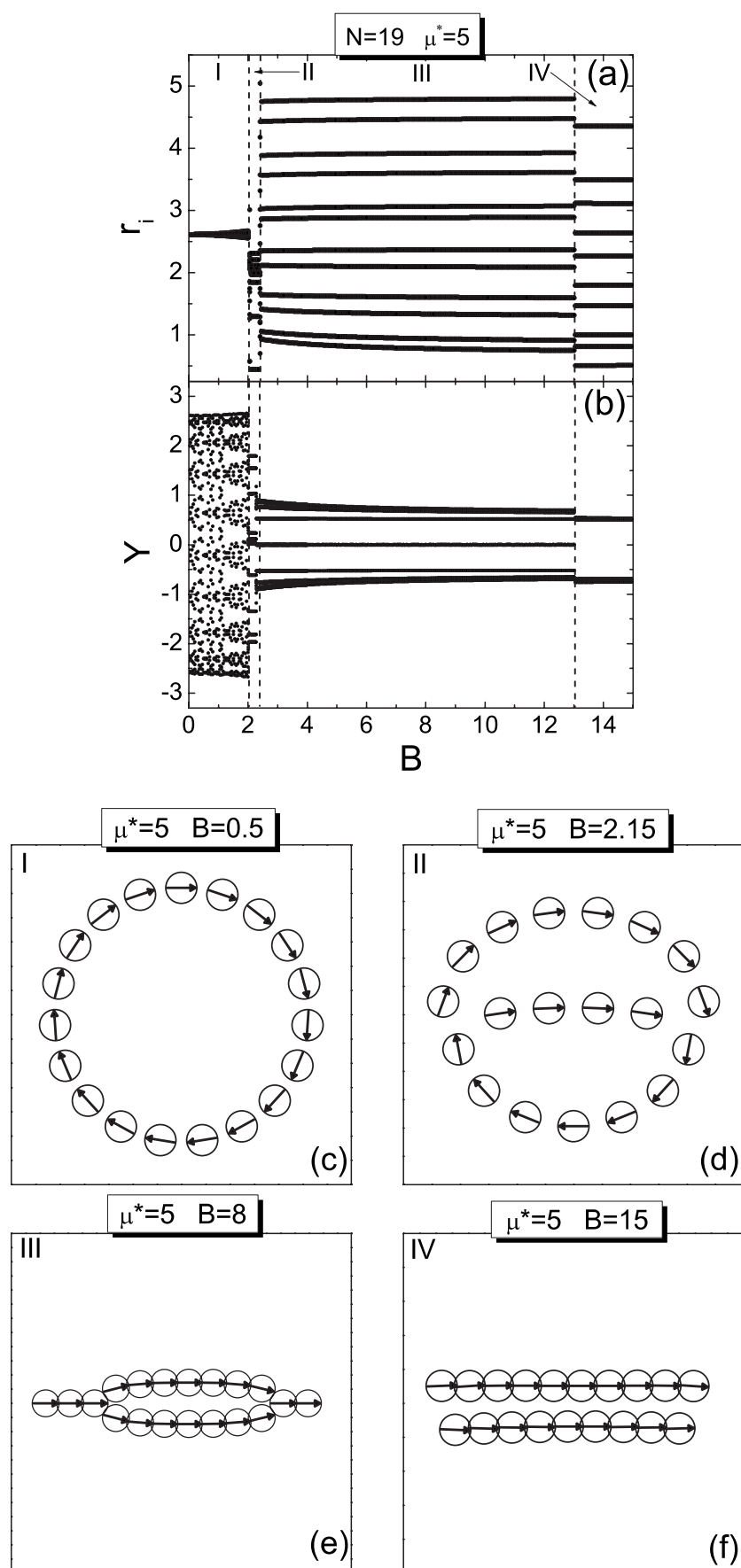

(d)

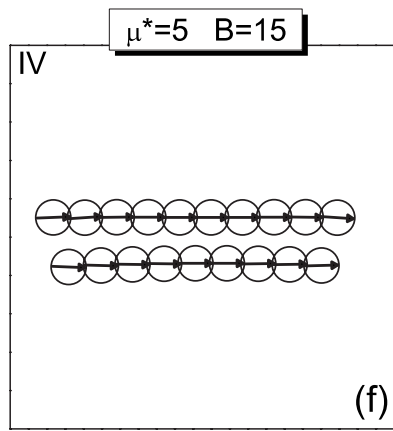

FIG. 7. (a) Radial position and (b) distance of each particle with respect to the $x$ axis of $N=19$ dipolar particles as a function of the magnetic field $B$ for fixed dipole moment $\mu^{*}=5$. (c)-(f) The ground state configuration of the system in different regions defined in (a).

shows that the energy necessary to excite the mode associated with the rotation of the whole system increases when the magnetic field is increased. This is reasonable since the increase of magnetic field decreases the circular symmetry of the system.

In order to better understand the influence of the magnetic field on the normal modes of the system we plot in Fig. 8(b) the lowest nonzero normal-mode frequency as a function of the magnetic field $B$. New subregions (IIIa and IIIb) separat-
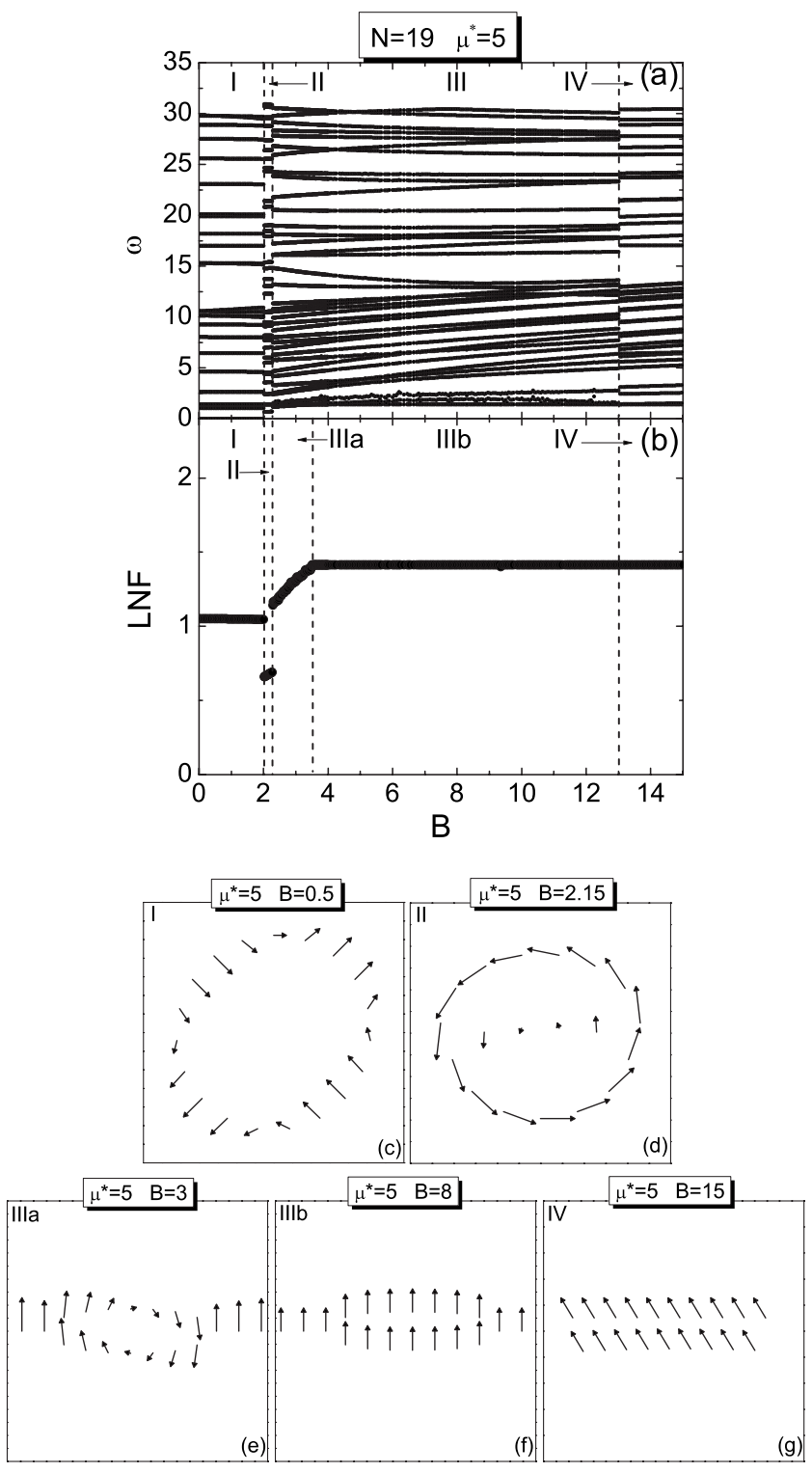

FIG. 8. (a) Normal-mode frequency and (b) lowest nonzero frequency (LNF) of $N=19$ dipolar particles as a function of magnetic moment $B$ for fixed dipole moment of particles $\mu^{*}=5$. (c) $-(\mathrm{g})$ Normal modes of vibration for each subregion defined in (b).

ing different $B$ intervals are defined in addition to the regions defined in Fig. 8(a). The vibrational motion of each particle in each region of Fig. 8(b) are shown in Figs. 8(c)-8(g). At regions IIIb and IV, the LNF corresponds to the mode associated with the motion of the center of mass. In addition, we observe that nontrivial configurations induce an interesting vibrational mode of particles such as, for example, the configuration corresponding to subregion IIIa [Fig. 7(e)]. In order to study the influence of the magnetic field on the angular oscillation of the particles, Figs. 9(a)-9(e) show the rotational component of the LNF mode for each region of Fig. 8(b). Notice that the system exhibits different rotational modes for each configuration. As observed for the vibrations, the rotational mode of the system in region III can be changed by increasing the value of $B$, leading the system to a scenario without rotation of particles [Fig. 9(d)] which is 


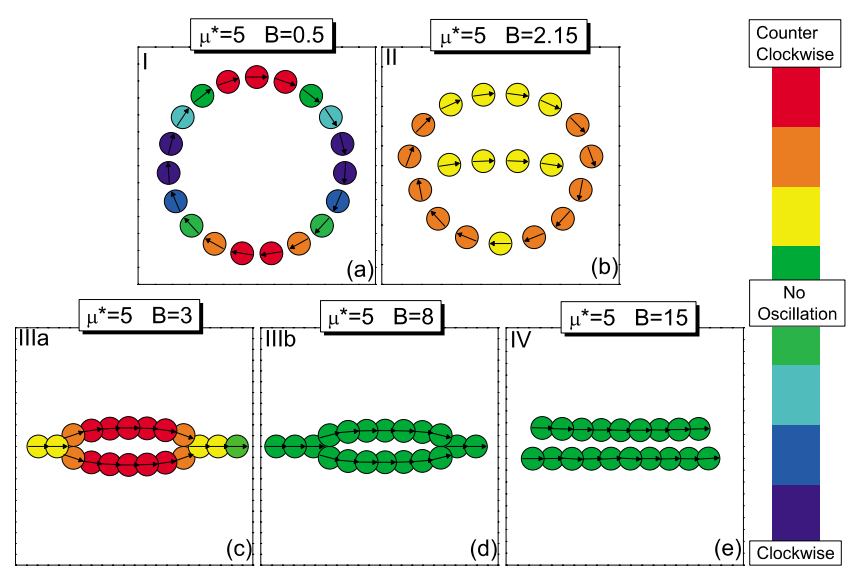

FIG. 9. (Color online) Normal modes of rotation for the system with $N=19$ particles and $\mu^{*}=5$. In each figure the subregions defined in Fig. 8(b) are indicated. The color scale gives the amplitude and the direction of rotational oscillation of particles.

associated with the frequency of the center of mass [see Fig. 8(b)]. This fact shows the strong dependence of the modes on the magnetic field $B$.

\section{Dependence on the number of particles}

Up to now, we have considered the specific example of a system composed of $N=19$ particles. One may wonder how general the conclusions are for systems with different numbers of particles. Therefore, we present now a study of the structure and normal frequencies of the system as a function of the number of particles $N$ for fixed values of the dipole moment $\mu^{*}$ and the magnetic field $B$.

\section{Zero magnetic field}

In order to study the self-assembled structure of the system of dipole particles, Fig. 10(a) shows the radial position of the particles in the ground state configuration of systems with different numbers of particles $N$ for $\mu^{*}=5$ and $B=0$. Figure 10(a) is divided into regions where the system exhibits diverse structures shown in Figs. 10(c)-10(e). As can be observed, region I of Fig. 10(a) is characterized by systems which present a kind of ferromagnetic configuration, i.e., all particles are aligned in the absence of a magnetic field [Fig. $10(\mathrm{c})]$. The arrangement of the particles in a line appears as the ground state configuration for systems up to $N=4$ particles (region I). The ring configuration is found in region II, i.e., for $5 \leqslant N \leqslant 20$ particles. It is important to note that the late appearance of the ring configuration is due to the fact that the energy between two dipole particles is minimum when they are aligned. The critical value $N=5$, for which the ring structure is obtained, increases with increasing $\mu^{*}$. In the present case, the line configuration is destroyed because the parabolic confinement folds the chain of particles into a closed line. The interparticle potential between the dipoles also influences the ring configuration which becomes more stable than in a system of charged particles. Indeed, we found that the single ring configuration is the ground state up to $N=20$ particles $(N=5$ particles in the case of charged par-
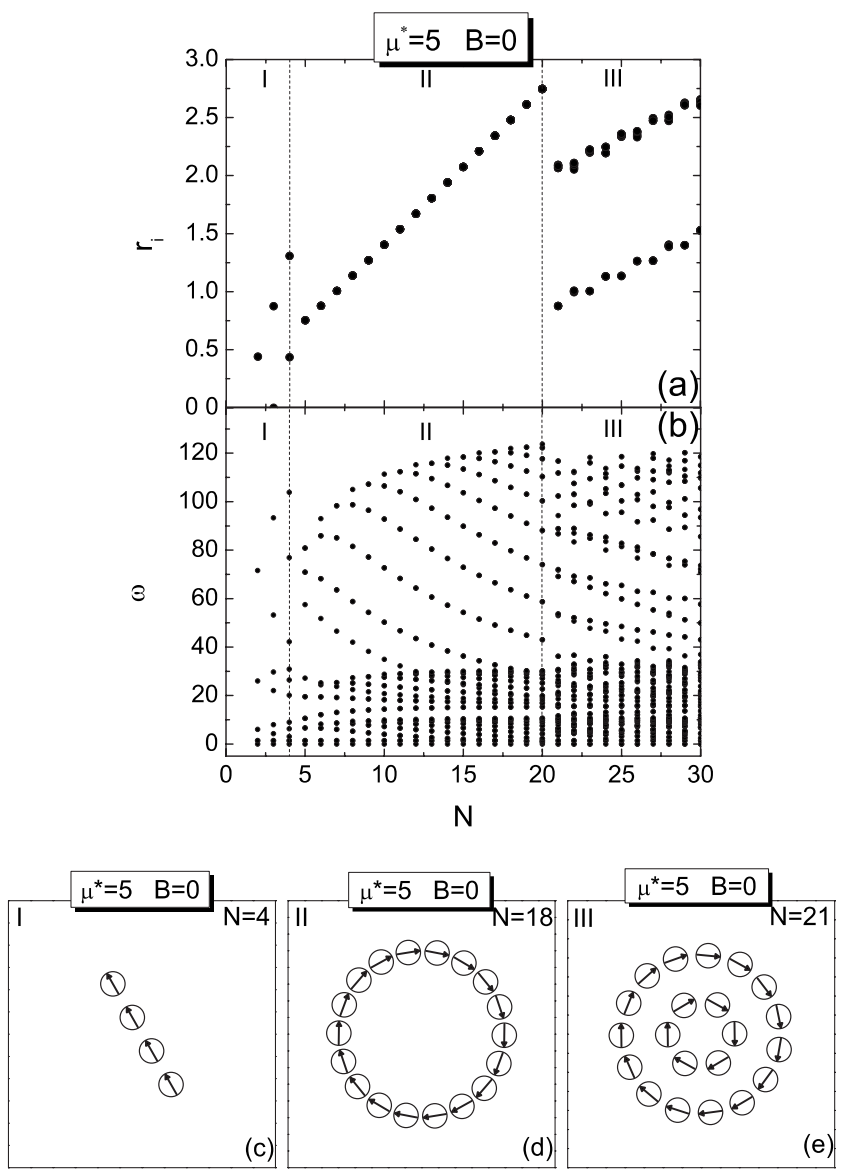

FIG. 10. (a) Radial position and (b) normal-mode frequency as a function of the number of particles $N$ for $\mu^{*}=5$ and $B=0$. (c)-(e) The ground state configuration of the system for the three regions defined in (a).

ticles [32]). For $N=21$ (region III), a second ring of particles shows up, forming a structure of two rings $(6,15)$ meaning that there are six particles in the inner shell and 15 particles in the outer one [see Fig. 10(e)].

The normal-mode spectrum is presented in Fig. 10(b) as a function of $N$. As can be observed, the frequencies exhibit jumps when the configuration changes discontinuously. In addition, we observe that the highest value of frequency is reached for a system with only a few particles and it does not increase further with $N$. Thus, new frequencies arise with values below the highest one, which is opposite to the case of the charged particle system where the highest frequency increases as a function of the number of particles [27].

\section{In the presence of a magnetic field}

In the following, systems with different numbers of particles $N$ are studied in the case of a nonzero magnetic field $B$. The radial position as a function of $N$ for $\mu^{*}=5$ and $B=5$ is shown in Fig. 11(a). For $2 \leqslant N \leqslant 12$ (region I), the particles are ordered into a single chain along the direction of $\mathbf{B}$ [Fig. 11(d)]. Note that the line structure is the ground state configuration for clusters with larger numbers of particles than those in the zero-magnetic-field case. This indicates that the number of particles in this structure increases with the mag- 

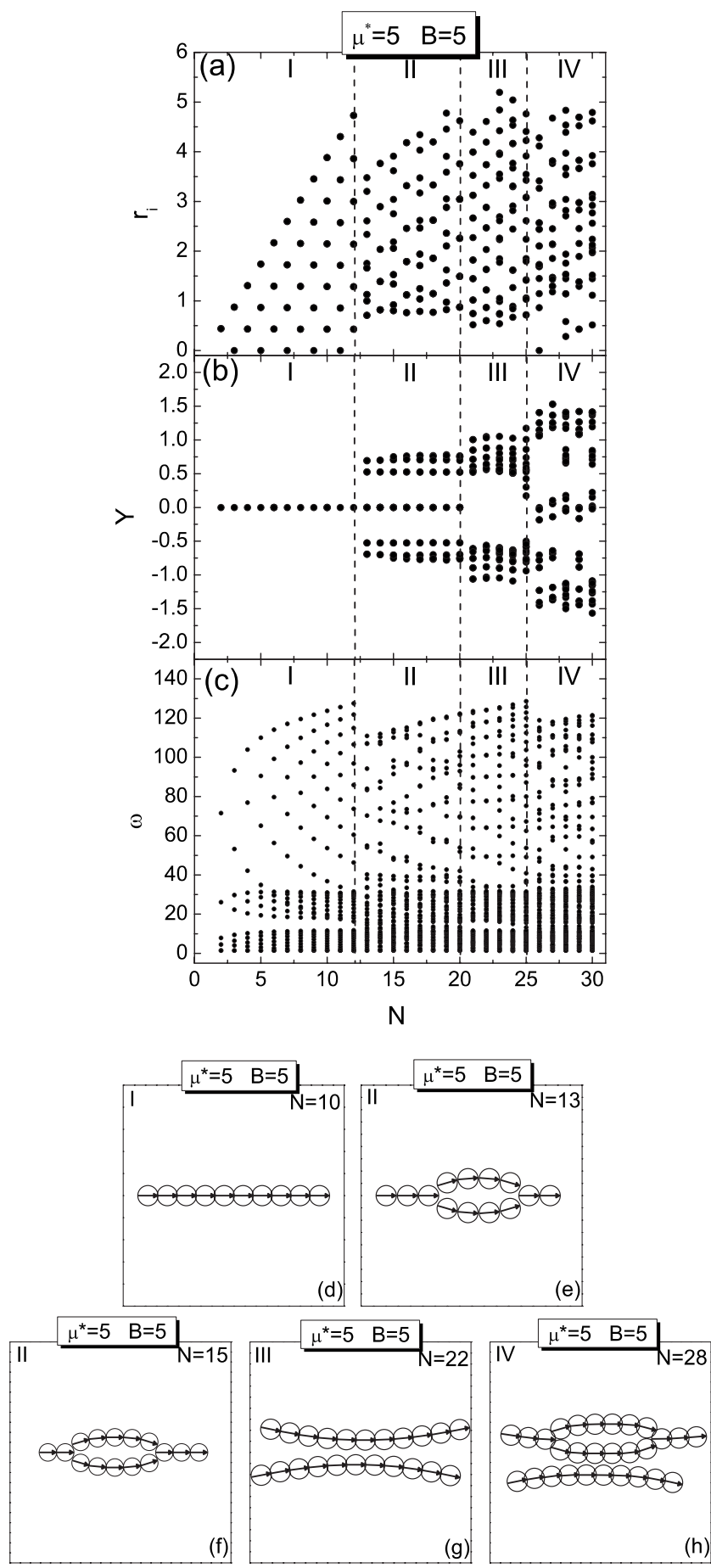

FIG. 11. (a) Radial position, (b) distance of each particles with respect to the $x$ axis, and (c) normal-mode frequency as a function of the number of particles for $\mu^{*}=5$ and $B=5$. (d)-(h) The ground state configuration of the system for each region defined in (a).

netic field, which stabilizes them in a line shape. When the system is composed of $N=13$ particles (region II), we find a 2 Y-shaped configuration which is observed for systems up to $N=20$ particles. Figures 11(e) and 11(f) present this structure for clusters with $N=13$ and 15 particles, respectively. In region III $(21 \leqslant N \leqslant 25)$, the system consists of two chains [see Fig. $11(\mathrm{~g})]$. Systems with $25<N \leqslant 30$ (region IV) particles consist of a mixture of a chain and a 2 Y-shaped configuration which can be observed in Fig. 11(h).

Although the system is being confined by a circular potential, we found that the flux-closure configurations (e.g., rings) do not appear for any number of particles of the dipole system in the presence of a magnetic field and $\mu^{*}=5$. This fact indicates that, in the case shown here, the magnetic field determines the configuration of the system, changing the symmetry of the system from circular to one that is parallel to the $x$ axis. In addition, we found that the magnetic field also influences the appearance of the Y-shaped configuration which was observed only in systems in the presence of a magnetic field. It is important to note that the closed Y-shaped configuration precedes the chain structure with a larger number of chains when the number of particles is increased. In other words, we observe that the closed Y-shaped configuration is preferentially formed before a new chain of particles. Therefore, we can conclude that the closed Y-shaped configuration arises due to the increase of the density of particles in a chain, which disappears when a new chain is formed.

Figure 11(c) shows the frequency spectrum as a function of the number of particles $N$. The qualitative dependence of the frequencies on $N$ in the presence of a magnetic field is similar to that of the system with $B=0$, i.e., the highest value of the frequency is reached for a system with few particles, and new frequencies appear for a larger number of particles below this value. We found in the previous section that most frequencies increase as a function of $\mu^{*}$ and remain practically unaltered as a function of $B$. Therefore, we conclude that the behavior of the frequencies in Figs. 10(b) and 11(c) is a consequence of the value of the dipole moment of the particles and therefore it is rather similar in a system with or without an applied magnetic field.

\section{CONCLUSION}

Systems consisting of a finite number of magnetic dipoles were analyzed as a function of the dipole moment of the particles and the magnitude of an external magnetic field applied parallel to the plane of the particles. The system of dipole particles undergoes several structural phase transitions as a function of the dipole moment and/or the magnetic field. In particular, when the dipole moment of the particles is increased at zero magnetic field, the hexagonal structure, which is the ground state configuration of a system of particles with zero dipole moment, was deformed in order to increase the structure symmetry, i.e., particles organize in rings when the dipole moment of the particles increases. When a magnetic field is applied, several different kinds of configurations are found as a function of the dipole moment of the particles like, for example, chains and Y-shaped structures, which were previously also encountered in experiments on infinite systems.

We found that the system under the influence of a magnetic field changes its circular to line symmetry, showing that the dipole-dipole interaction in a magnetic field dominates over the confinement potential. In addition, the system exhibits a special configuration, namely, a closed Y-shaped 
structure which occurs when two Y-shaped configurations are connected. This structure is attributed to the high density in one direction due to the balance between the confinement potential and the magnetic field. The dependence on the magnetic field was also studied. The system presented an interesting mixture of chain and ring structures for some values of the magnetic field.

The normal-mode frequencies were also studied as a function of the dipole moment and/or magnetic field. The vibrational and rotational oscillation of particles, associated with the lowest nonzero frequency, were studied in detail. In the case of zero magnetic field, all frequencies, except those of the center of mass and rotation modes of the system, increase as a function of the dipole moment of particles. In addition, it was found that the behavior of the LNF is also determined by the rotational motion of the particles and the central particle of the hexagonal structure exhibits the highest amplitude of rotation. Therefore, the rotational motion of particles was shown to be very important in determining the behavior of the LNFs and as a consequence in predicting the stability of the system. In the presence of a magnetic field, it was found that, in addition to most of the frequencies, the frequency of rotation of the system also increases as a function of the dipole moment of the particles. In this case, only the frequency associated with the normal mode of the center of mass remains constant. It was observed that the magnetic field induces a chain structure but produces no effect on the direction of oscillation of the particles. In the hexagonal structure, the particles with the highest amplitude of rotational oscillation are located at the edge of the system. When the magnetic field is varied, the frequencies remain practically unaltered except the one associated with the rotation of the whole system, which changes its value as a function of the magnetic field.

The dependence on the number of particles was also investigated. In the zero-magnetic-field case, the system with few number of particles $(N<5)$ showed a stable line configuration. In addition, in a dipolar system the maximum number of particles in a single ring $\left(N=20\right.$ when $\left.\mu^{*}=5\right)$ was found to be larger than that for Coulomb interparticle interaction $(N=5)$. Systems with a large number of particles $(20$ $<N<30$ for $\mu^{*}=5$ ) show a structure composed of two rings. In the nonzero-magnetic-field case, the system exhibits chains that are more stable and therefore they can hold a larger number of particles $\left(N=12\right.$ for $\mu^{*}=5$ and $\left.B=5\right)$. Due to the fact that the magnetic field acts also as a unidirectional confinement potential, ring structures were not observed.

\section{ACKNOWLEDGMENTS}

F.F.M., W.P.F., and G.A.F. were supported by the Brazilian National Research Councils CNPq and CAPES and the Ministry of Planning (FINEP). Part of this work was supported by the Flemish Science Foundation (FWO-Vl) and the bilateral program between Flanders and Brazil.
[1] Q. A. Pankhurst, J. Connolly, S. K. Jones, and J. Dobson, J. Phys. D 36, R167 (2003).

[2] G. Cheng, D. Romero, G. T. Fraser, and A. R. H. Walker, Langmuir 21, 12055 (2005).

[3] A. Yethiraj and A. Van Blaaderen, Nature (London) 421, 513 (2003).

[4] A. S. Lubbe, C. Alexiou, and C. Bergemann, J. Surg. Res. 95, 200 (2001).

[5] A. Yu. Zubarev and L. Yu. Iskakova, Physica A 376, 38 (2007).

[6] K. Raj and R. Moskowitz, J. Magn. Magn. Mater. 85, 233 (1990).

[7] K. Raj, B. Moskowitz, and R. Casciari, J. Magn. Magn. Mater. 149, 174 (1995).

[8] P. D. Duncan and P. J. Camp, Phys. Rev. Lett. 97, 107202 (2006).

[9] M. Klokkenburg, B. H. Erne, A. Wiedenmann, A. V. Petukhov, and A. P. Philipse, Phys. Rev. E 75, 051408 (2007).

[10] L. Assoud, R. Messina, and H. Löwen, Europhys. Lett. 80, 48001 (2007).

[11] V. F. Puntes, K. M. Krishnan, and A. P. Alivisatos, Science 291, 2115 (2001).

[12] K. Butter, P. H. H. Bomans, P. M. Frederik, G. J. Vroege, and A. P. Philipse, Nat. Mater. 2, 88 (2003).

[13] K. Butter, P. H. Bomans, P. M. Frederik, G. J. Vroege, and A. P. Philipse, J. Phys.: Condens. Matter 15, S1451 (2003).

[14] M. Klokkenburg, R. P. A. Dullens, W. K. Kegel, B. H. Erne, and A. P. Philipse, Phys. Rev. Lett. 96, 037203 (2006).
[15] P. D. Duncan and P. J. Camp, J. Chem. Phys. 121, 11322 (2004).

[16] K. Van Workum and J. F. Douglas, Phys. Rev. E 73, 031502 (2006).

[17] E. M. Claesson, B. H. Erne, and A. P. Philipse, J. Phys.: Condens. Matter 19, 286102 (2007).

[18] W. P. Ferreira, F. F. Munarin, K. Nelissen, R. N. Costa Filho, F. M. Peeters, and G. A. Farias, Phys. Rev. E 72, 021406 (2005).

[19] F. F. Munarin, W. P. Ferreira, G. A. Farias, and F. M. Peeters, Phys. Rev. B 76, 035336 (2007).

[20] M. Klindworth, A. Melzer, A. Piel, and V. A. Schweigert, Phys. Rev. B 61, 8404 (2000); W.-T. Juan, Z.-H. Huang, J.-W. Hsu, Y.-J. Lai, and L. I, Phys. Rev. E 58, R6947 (1998).

[21] A. Melzer, M. Klindworth, and A. Piel, Phys. Rev. Lett. 87, 115002 (2001); A. Melzer, Phys. Rev. E 67, 016411 (2003).

[22] R. Bubeck, P. Leiderer, and C. Bechinger, Europhys. Lett. 60, 474 (2002).

[23] W. P. Ferreira, F. F. Munarin, G. A. Farias, and F. M. Peeters, J. Phys.: Condens. Matter 18, 9385 (2006).

[24] L. Baraban, D. Makarov, M. Albrecht, N. Rivier, P. Leiderer, and A. Erbe, Phys. Rev. E 77, 031407 (2008).

[25] R. Bubeck, C. Bechinger, S. Neser, and P. Leiderer, Phys. Rev. Lett. 82, 3364 (1999).

[26] I. V. Schweigert, V. A. Schweigert, and F. M. Peeters, Phys. Rev. Lett. 84, 4381 (2000).

[27] V. A. Schweigert and F. M. Peeters, Phys. Rev. B 51, 7700 (1995). 
[28] M. Klokkenburg, B. H. Erne, J. D. Meeldijk, A. Wiedenmann, A. V. Petukhov, R. P. A. Dullens, and A. P. Philipse, Phys. Rev. Lett. 97, 185702 (2006).

[29] B. Partoens and F. M. Peeters, J. Phys.: Condens. Matter 9, 5383 (1997).
[30] T. Tlusty and S. A. Safran, Science 90, 1328 (2000).

[31] A. Yu. Zubarev and L. Yu. Iskakova, Phys. Rev. E 76, 061405 (2007).

[32] V. M. Bedanov and F. M. Peeters, Phys. Rev. B 49, 2667 (1994). 